\title{
Explicit limit cycles of a class of Kolmogorov system
}

\author{
Salah Benyoucef $^{\mathrm{a}^{*}}$ and Ahmed Bendjeddou ${ }^{\mathrm{b}}$

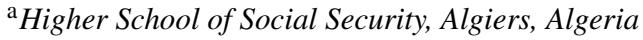 \\ ${ }^{\mathrm{b}}$ University of Setif 1, department of Mathematics, 19000 Algeria \\ ${ }^{*}$ Corresponding author E-mail: saben21@yahoo.fr
}

\section{Article Info}

Keywords: Kolmogorov differential system, Invariant curve, Periodic solution, Hyperbolic limit cycle 2010 AMS: $34 C 25,34 A 34,34 C 05$

Received: 18 May 2018

Accepted: 16 September 2018

Available online: 30 September 2018

\begin{abstract}
A class of Kolmogorov differential system is introduced. It is shown that under suitable assumptions on degrees and parameters, algebraic limit cycles can occur. we propose an easy algorithm to test the existence of limit cycles and we give them explicit expressions.
\end{abstract}

\section{Introduction}

The second part of sixteenth problem of Hilbert rases the question of the maximum number and the mutual position of limit cycles of the differential system:

$$
\left\{\begin{array}{l}
\dot{x}=\frac{d x}{d t}=P(x, y) \\
\dot{y}=\frac{d y}{d t}=Q(x, y)
\end{array}\right.
$$

where $P$ and $Q$ are two polynomials of any degree.

Many mathematical models in population dynamics, frequently involve the systems of ordinary differential equations having the form

$$
\left\{\begin{array}{c}
\dot{x}=\frac{d x}{d t}=x F(x, y), \\
\dot{y}=\frac{d y}{d t}=y G(x, y),
\end{array}\right.
$$

$x(t)$ and $y(t)$ represent the population density of two species at time $t$, and $F(x, y), G(x, y)$ are the capita growth rate of each specie, usually, such systems are called Kolmogorov systems.

Kolmogorov models are widely used in ecology to describe the interaction between two populations, and a limit cycle corresponds to an equilibrium state of the system.

In mathematical modeling of ecological systems and population dynamics, more mathematicians and scientists were attracted to the subject and several results have been published, May[15], Kuang and Freedman[12] , X. Huang, Y. Wang, A.Cheng [11], and others.

When $F(x, y)$ and $G(x, y)$ are polynomials of degrees $\geq 2$, limit cycles can occur and there is an extensive literature dealing with their existence, number and stability (see for instance Lloyd, Pearson, Sáez and Szántó[13], X.C. Huang, L. Zhu [9], X.C.Huang[10], S.Boqian and L.Demeng [5], K.S. Cheng [6],... ), but to our knowledge, the exact analytic expressions of the limit cycles for a given kolmogorov system is still unknown except for simple and specific cases. This paper is a contribution in this direction, to determine the number of limit cycles and to give their explicit form.

Motivated by the recent publication of some research papers exhibiting planar polynomial systems with one or more algebraic limit cycles analytically given (see for instance A. Bendjeddou and R. Cheurfa[1], [2], S.Benyoucef, A, Barbach and A.Bendjeddou, [3], S. Benyoucef 
and A.Bendjeddou[4], S.Chengbin, S.Boqian[7], Lloyd, Pearson, Sáez and Szántó[14], Peng Yue-hui[17]), we will study the existence and the number of limit cycles of a class of Kolmogorov system, and give their explicit form.

Mainly based on the work of Article[4], we will enter some disruption on the differential system, adding other terms and changing the parameters.

\section{Some useful notions}

Let recall some useful notions.

For $U \in \mathbb{R}[x, y]$, the algebraic curve $U=0$ is called an invariant curve of the polynomial system (1.2), if for some polynomial $K \in \mathbb{R}[x, y]$ called the cofactor of the algebraic curve, we have

$$
x F(x, y) \frac{\partial U}{\partial x}+y G(x, y) \frac{\partial U}{\partial y}=K U
$$

The curve $\Gamma=\left\{(x, y) \in \mathbb{R}^{2}: U(x, y)=0\right\}$ is nonsingular of system (1.2) if it is without singular points. The singular points or the equilibrium points of system (1.2) satisfy

$$
\left\{\begin{array}{l}
x F(x, y)=0 \\
y G(x, y)=0
\end{array}\right.
$$

If the curve $\Gamma$ is nonsingular of system (1.2), the equilibrium points of the system are contained either in its unbounded components or are located on the curve $K=0$.

A limit cycle $\gamma=\{(x(t), y(t)), t \in[0, T]\}$, is a $T$-periodic solution isolated with respect to all other possible periodic solutions of the system.

The $T$-periodic solution $\gamma$ is an hyperbolic limit cycle if $\int_{0}^{T} \operatorname{div}(\gamma(t)) d t$ is different from zero [16].

We construct here a multi-parameter planar differential system admitting the components of curve

$$
\Gamma=\left\{(x, y) \in \mathbb{R}^{2}: x^{2 n}+b x^{n}+c y^{2 m}+y^{m}\left(d+f x^{n}\right)+h=0,(n, m) \in \mathbb{N}^{*} \times \mathbb{N}^{*}\right\}
$$

as hyperbolic limit cycles if some conditions on the parameters are satisfied.

Note that our study is not restricted to the realistic quadrant $\left\{(x, y) \in \mathbb{R}^{2}: x>0, y>0\right\}$, but it covers all the domain $\mathbb{R}^{2}$.

\section{The main result}

As a main result, we have the following theorem

Theorem 3.1. The system

$$
\left\{\begin{array}{l}
\dot{x}=x\left((y P(x)+V(y))\left(a x^{2 n}+b x^{n}+c y^{2 m}+d y^{m}+f x^{n} y^{m}+h\right)-m y^{m}\left(d+2 c y^{m}+f x^{n}\right)\right) \\
\dot{y}=y\left((x Q(y)+W(x))\left(a x^{2 n}+b x^{n}+c y^{2 m}+d y^{m}+f x^{n} y^{m}+h\right)+n x^{n}\left(b+2 a x^{n}+f y^{m}\right)\right)
\end{array}\right.
$$

where $a, c$ are positive real, $b, d$ are negative real, $f$ is negative real such as $f^{2}<4 a c, h$ satisfied

$$
\max \left\{\frac{b^{2}}{4 a}, \frac{d^{2}}{4 c}\right\}<h<\frac{b d f-b^{2} c-a d^{2}}{f^{2}-4 a c},(n, m) \in \mathbb{N}^{*} \times \mathbb{N}^{*}
$$

$P(x)$ and $Q(y)$ are odd polynomial functions of any degree with positive coefficients, $V(x)$ and $W(y)$ are analytic functions.

This system admits

One limit cycle when $n$ and $m$ are odd numbers.

Two limit cycles when one of numbers $n$ and $m$ is odd and the other is even.

Four limit cycles when $n$ and $m$ are even numbers .

The limit cycles are hyperbolics represented by the curve $\Gamma$.

Proof. We will prove that $\Gamma$ is nonsingular composed of ovals and it is an invariant curve of system (3.1), and $\int_{0}^{T} d i v(\Gamma) d t \neq 0$. (see for instance Perko [16])

i) The curve $\Gamma$ is non singular of system composed of ovals.

We recall that the curve $\Gamma$ is non-singular of system (3.1) if the following system has no real solution.

$$
\left\{\begin{array}{c}
a x^{2 n}+b x^{n}+c y^{2 m}+d y^{m}+f x^{n} y^{m}+h=0 \\
n y x^{n}\left(b+2 a x^{n}+f y^{m}\right)=0 \\
-m x y^{m}\left(d+2 c y^{m}+f x^{n}\right)=0
\end{array}\right.
$$

Note that the curve $\Gamma$ does not intersect the axes, $c y^{2 m}+d y^{m}+h \neq 0$ because $h>\frac{d^{2}}{4 c}$, and $a x^{2 n}+b x^{n}+h \neq 0$ because $h>\frac{b^{2}}{4 a}$, then the possible critical points on $\Gamma$ are: $A_{1}\left(-\sqrt[n]{\frac{2 b c-d f}{f^{2}-4 a c}},-\sqrt[m]{\frac{2 a d-b f}{f^{2}-4 a c}}\right), A_{2}\left(-\sqrt[n]{\frac{2 b c-d f}{f^{2}-4 a c}}, \sqrt[m]{\frac{2 a d-b f}{f^{2}-4 a c}}\right), A_{3}\left(\sqrt[n]{\frac{2 b c-d f}{f^{2}-4 a c}},-\sqrt[m]{\frac{2 a d-b f}{f^{2}-4 a c}}\right)$, $A_{4}\left(\sqrt[n]{\frac{2 b c-d f}{f^{2}-4 a c}}, \sqrt[m]{\frac{2 a d-b f}{f^{2}-4 a c}}\right)$ when $m$ and $n$ are even numbers.

$A_{4}$ when $m$ and $n$ are odd numbers.

$A_{2}, A_{4}$ when $m$ is odd and $n$ is even. 
$A_{3}, A_{4}$ when $m$ is even and $n$ is odd.

As $h \neq \frac{1}{f^{2}-4 a c}\left(b d f-a d^{2}-b^{2} c\right)$ and $f^{2} \neq 4 a c$, then all the points $A_{1}, A_{2}, A_{3}, A_{4}$ are not on $\Gamma$, and the curve $\Gamma$ is nonsingular.

Now, we prove that $\Gamma$ is composed by ovals.

We consider the equation (2.3) as

$$
c z^{2}+\left(d+f x^{n}\right) z+\left(a x^{2 n}+b x^{n}+h\right)=0 \text { where } z=y^{m}
$$

The discriminant $\Delta=\left(f^{2}-4 a c\right) s^{2}+2(d f-2 b c) s+d^{2}-4 c h$ where $s=x^{n}$ $\Delta_{\Delta}^{\prime}=4 b^{2} c^{2}-4 b c d f-16 a h c^{2}+4 a c d^{2}+4 h c f^{2}$

We note that if $h>\frac{b^{2} c^{2}-b d f+a d^{2}}{4 a c-f^{2}}, \Delta_{\Delta}^{\prime}<0$, and as $f^{2}<4 a c$ then $\Delta<0$, and there is no real solution of equation (2.3).

If $h=\frac{b^{2} c^{2}-b d f+a d^{2}}{4 a c-f^{2}}, \Delta_{\Delta}^{\prime}=0$, and $\Delta=\left(f^{2}-4 a c\right)\left(s-\frac{2 b c-d f}{f^{2}-4 a c}\right)^{2}<0$, with $s \neq \frac{2 b c-d f}{f^{2}-4 a c}$, and there is no real solution of equation (2.3).

If $h<\frac{b^{2} c^{2}-b d f+a d^{2}}{4 a c-f^{2}}, \Delta_{\Delta}^{\prime}>0$ and we have

$$
\begin{aligned}
& s_{1}=\frac{1}{4 a c-f^{2}}\left(2 \sqrt{b^{2} c^{2}-4 a h c^{2}+a c d^{2}-b c d f+h c f^{2}}-2 b c+d f\right) \\
& s_{2}=\frac{1}{4 a c-f^{2}}\left(-2 \sqrt{b^{2} c^{2}-4 a h c^{2}+a c d^{2}-b c d f+h c f^{2}}-2 b c+d f\right)
\end{aligned}
$$

The expression

$$
\frac{1}{4 a c-f^{2}}\left(2 \sqrt{b^{2} c^{2}-4 a h c^{2}+a c d^{2}-b c d f+h c f^{2}}-2 b c+d f\right)
$$

is positive,

and if $\frac{d^{2}}{4 c}<h$, then the expression $\frac{1}{4 a c-f^{2}}\left(-2 \sqrt{b^{2} c^{2}-4 a h c^{2}+a c d^{2}-b c d f+h c f^{2}}-2 b c+d f\right)$ is also positive.

We distinguish the following cases

1. $n$ is odd number

The equation $\Delta=0$ admits two real solutions

$$
\begin{aligned}
& x_{1}=\sqrt[n]{\frac{1}{4 a c-f^{2}}\left(2 \sqrt{b^{2} c^{2}-4 a h c^{2}+a c d^{2}-b c d f+h c f^{2}}-2 b c+d f\right)} \\
& x_{2}=\sqrt[n]{\frac{1}{4 a c-f^{2}}\left(-2 \sqrt{b^{2} c^{2}-4 a h c^{2}+a c d^{2}-b c d f+h c f^{2}}-2 b c+d f\right)}
\end{aligned}
$$

For $x \in\left[x_{1}, x_{2}\right]$ the discriminant $\Delta$ is positive and the equation (2.3) admits two real solutions

$$
\begin{aligned}
& z_{1}=\frac{-\left(d+f x^{n}\right)-\sqrt{\left(f^{2}-4 a c\right) x^{2 n}+2(d f-2 b c) x^{n}+d^{2}-4 c h}}{2 c} \\
& z_{2}=\frac{-\left(d+f x^{n}\right)+\sqrt{\left(f^{2}-4 a c\right) x^{2 n}+2(d f-2 b c) x^{n}+d^{2}-4 c h}}{2 c}
\end{aligned}
$$

As $c>0$ and $h>\frac{b^{2}}{4 a}, z_{1}, z_{2}$ are positive.

If $m$ is odd number, they are two real solutions of equation (2.3) that depend on $y$ which are

$$
\begin{aligned}
& y_{1}=\sqrt[m]{\frac{-\left(d+f x^{n}\right)-\sqrt{\left(f^{2}-4 a c\right) x^{2 n}+2(d f-2 b c) x^{n}+d^{2}-4 c h}}{2 c}} \\
& y_{2}=\sqrt[m]{\frac{-\left(d+f x^{n}\right)+\sqrt{\left(f^{2}-4 a c\right) x^{2 n}+2(d f-2 b c) x^{n}+d^{2}-4 c h}}{2 c}}
\end{aligned}
$$

$x \rightarrow y_{1}$ is decreasing function when $\left.x \in\right] x_{1}, x_{0}$ [ and an increasing function when $\left.x \in\right] x_{0}, x_{2}$ [ $x \rightarrow y_{2}$ is an increasing function when $\left.x \in\right] x_{1}, x_{0}^{\prime}[$ and a decreasing function when $x \in] x_{0}^{\prime}, x_{2}[$ 
where $x_{0}=\sqrt[n]{-\frac{1}{a\left(-4 a c+f^{2}\right)}\left(f \sqrt{a^{2} d^{2}+a b^{2} c-4 a^{2} c h+a f^{2} h-a b d f}-2 a b c+a d f\right)}$

$$
x_{0}^{\prime}=\sqrt[n]{\frac{1}{a\left(-4 a c+f^{2}\right)}\left(f \sqrt{a^{2} d^{2}+a b^{2} c-4 a^{2} c h+a f^{2} h-a b d f}+2 a b c-a d f\right)}
$$

on the other hand

$$
\begin{aligned}
& y_{1}\left(x_{1}\right)=y_{2}\left(x_{1}\right)=\sqrt[m]{\frac{1}{c\left(f^{2}-4 a c\right)}\left(f \sqrt{b^{2} c^{2}-b c d f-4 a h c^{2}+a c d^{2}+h c f^{2}}+2 a c d-b c f\right)} \\
& y_{1}\left(x_{2}\right)=y_{2}\left(x_{2}\right)=\sqrt[m]{-\frac{1}{c\left(f^{2}-4 a c\right)}\left(f \sqrt{b^{2} c^{2}-b c d f-4 a h c^{2}+a c d^{2}+h c f^{2}}-2 a c d+b c f\right)}
\end{aligned}
$$

then $\Gamma$ is composed of an oval in the area $D_{1}=\left\{(x, y) \in \mathbb{R}^{2}, x_{1}<x<x_{2}, y_{1}\left(x_{0}\right)<y<y_{2}\left(x_{0}^{\prime}\right)\right\}$.

$D_{1}$ is in the realistic quadrant.

If $m$ is even number they are four real solutions of equation (2.3) that depend on $y$ which are $y_{1}, y_{2},-y_{1},-y_{2}$

with the same process as before, we conclude that the curve $\Gamma$ is composed of two ovals, one is in the area $D_{1}$ and the other is in the area $D_{2}=\left\{(x, y) \in \mathbb{R}^{2}, x_{1} \leq x \leq x_{2},-y_{2}\left(x_{0}^{\prime}\right) \leq y \leq-y_{1}\left(x_{0}\right)\right\}$.

2. $n$ is even number

The equation $\Delta=0$ admits four real solutions $x_{1}, x_{2},-x_{1},-x_{2}$

For $x \in\left[-x_{2},-x_{1}\right] \cup\left[x_{1}, x_{2}\right]$ the discriminant $\Delta$ is positive and the equation (2.3) admits two real solutions $z_{1}, z_{2}$.

If $m$ is odd number, they are two real solutions of equation (2.3) that depend on $y$ wich are

$$
\begin{aligned}
& y_{1}=\sqrt[m]{\frac{-\left(d+f x^{n}\right)-\sqrt{\left(f^{2}-4 a c\right) x^{2 n}+2(d f-2 b c) x^{n}+d^{2}-4 c h}}{2 c}}, \\
& y_{2}=\sqrt[m]{\frac{-\left(d+f x^{n}\right)+\sqrt{\left(f^{2}-4 a c\right) x^{2 n}+2(d f-2 b c) x^{n}+d^{2}-4 c h}}{2 c}}
\end{aligned}
$$

If $m$ is even number, they are four real solutions of equation (2.3) that depend on $y$

$$
y_{1}, y_{2},-y_{1},-y_{2}
$$

As before, we conclude that, when $m$ is odd number, the curve $\Gamma$ is composed of two ovals, one is in the area $D_{1}$, and the other is in the area $D_{3}=\left\{(x, y) \in \mathbb{R}^{2},-x_{2} \leq x \leq-x_{1}, y_{1}\left(-x_{0}\right) \leq y \leq y_{2}\left(-x_{0}^{\prime}\right)\right\}$.

When $m$ is even number, the curve $\Gamma$ is composed of four ovals in the areas $D_{1}, D_{2}, D_{3}, D_{4}$

$D_{4}=\left\{(x, y) \in \mathbb{R}^{2},-x_{2} \leq x \leq-x_{1},-y_{2}\left(-x_{0}^{\prime}\right) \leq y \leq-y_{1}\left(-x_{0}\right)\right\}$

ii) $\Gamma$ is an invariant curve of system (3.1)

$$
\frac{\partial U}{\partial x} \dot{x}+\frac{\partial U}{\partial y} \dot{y}=\left(n x^{n}(y P(x)+V(y))\left(b+2 a x^{n}+f y^{m}\right)+m y^{m}(x Q(y)+W(x))\left(d+2 c y^{m}+f x^{n}\right)\right) U
$$

the cofactor is

$$
K(x, y)=n x^{n}(y P(x)+V(y))\left(b+2 a x^{n}+f y^{m}\right)+m y^{m}(x Q(y)+W(x))\left(d+2 c y^{m}+f x^{n}\right)
$$

iii) $\int_{0}^{T} \operatorname{div}(\Gamma) d t \neq 0$

Note that

$$
\int_{0}^{T} \operatorname{div}(\Gamma) d t=\int_{0}^{T} K(x(t), y(t)) d t
$$

see for instance Giacomini \& Grau[8].

$$
\begin{aligned}
\int_{0}^{T} K(x(t), y(t)) d t & =\int_{0}^{T} n x^{n}(y P(x)+V(y))\left(b+2 a x^{n}+f y^{m}\right) d t \\
& +\int_{0}^{T} m y^{m}(x Q(y)+W(x))\left(d+2 c y^{m}+f x^{n}\right) d t \\
& =\oint_{\Gamma} \frac{n x^{n}(y P(x)+V(y))\left(b+2 a x^{n}+f y^{m}\right)}{n y x^{n}\left(b+2 a x^{n}+f y^{m}\right)} d y-\oint_{\Gamma} \frac{m y^{m}(x Q(y)+W(x))\left(d+2 c y^{m}+f x^{n}\right)}{m x y^{m}\left(d+2 c y^{m}+f x^{n}\right)} d x \\
& =\oint_{\Gamma}\left(P(x)+\frac{V(y)}{y}\right) d y-\oint_{\Gamma}\left(Q(y)+\frac{W(x)}{x}\right) d x
\end{aligned}
$$




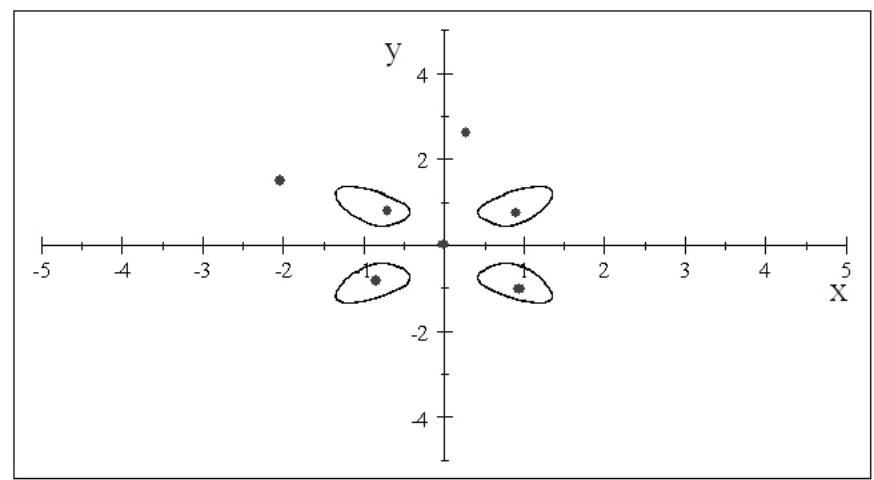

Figure 5.1: Limit cycles of system(5.1) with singular points

by applying the GREEN formula

$$
\oint_{\Gamma}\left(P(x)+\frac{V(y)}{y}\right) d y-\oint_{\Gamma}\left(Q(y)+\frac{W(x)}{x}\right) d x=\iint_{i n t(\Gamma)}\left(\frac{d(P(x))}{d x}+\frac{d(Q(y)}{d y}\right) d x d y
$$

where $\operatorname{int}(\Gamma)$ denotes the interior of $\Gamma$ (the region bounded by $\Gamma$ ).

As $P(x)$ and $Q(y)$ are odd polynomial functions with positive coefficients then $P^{\prime}(x)$ and $Q^{\prime}(y)$ are pair polynomial functions with all the coefficients are positive. We conclude that $\forall(x, y) \in$ int $(\Gamma),\left(P^{\prime}(x)+Q^{\prime}(y)\right)>0$ then $\int_{0}^{T} K(x(t), y(t)) d t$ is nonzero.

Remark 3.2. We can generalize Theorem 3.1 such $P(x)$ and $Q(y)$ are analytic functions, in this case we must add the condition $\forall(x, y) \in$ int $(\Gamma), P^{\prime}(x)+Q^{\prime}(y)$ is nonzero.

Remark 3.3. If the study is restricted to the realistic quadrant $\left\{(x, y) \in \mathbb{R}^{2}: x>0, y>0\right\}$, then the theorem remains true with odd degrees $n$ and $m$, in this case the system (3.1) admits one limit cycle in the realistic quadrant.

\section{Algorithm}

* Reading data of system (3.1)

Reading degrees $m$ and $n$ such that $m>0$ and $n>0$.

Reading coefficients $a, b, c, d, f, h$ such that $a>0, b<0, c>0, d<0, f<0$ and $f^{2}<4 a c$.

Enter the odd polynomial functions $P(x)$ and $Q(y)$ with positive coefficients.

Enter the analytic functions $V(x)$ and $W(y)$.

* Existence of limit cycles

If $\max \left\{\frac{b^{2}}{4 a}, \frac{d^{2}}{4 c}\right\}<h<\frac{b d f-b^{2} c-a d^{2}}{f^{2}-4 a c}$ then

if $m$ and $n$ are odd numbers there is one limit cycle

if $m$ and $n$ are even numbers there are four limit cycles

if one of numbers $m$ and $n$ is odd and the other is even there are two limit cycles

else

there is not limit cycle

endif

* Explicit form of limit cycle

when there exist limit cycle its explicit form is $x^{2 n}+b x^{n}+c y^{2 m}+y^{m}\left(d+f x^{n}\right)+h=0$

\section{Examples}

Example 5.1. Let $m=n=2, a=2, b=-2, c=2, d=-2, f=-2, h=1, P(x)=x, Q(y)=y, V(y)=y+1, W(x)=x-1$

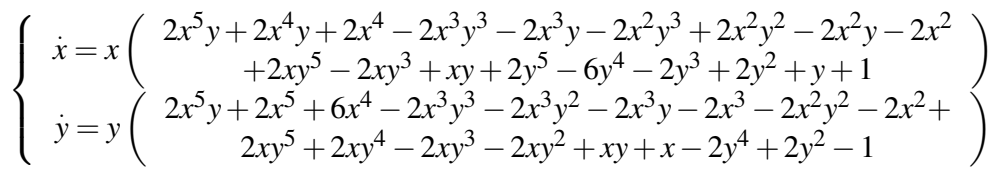

The system (5.1) admits four limit cycles represented by the curve $2 x^{4}-2 x^{2}+2 y^{4}+y^{2}\left(-2-2 x^{2}\right)+1=0$, and it has seven singular points, three are saddle points, two are stable focus and two are instable focus. The limit cycles in the first and third quadrant around stable focus and limit cycles in the second and fourth quadrant around unstable focus. Figure (5.1) 


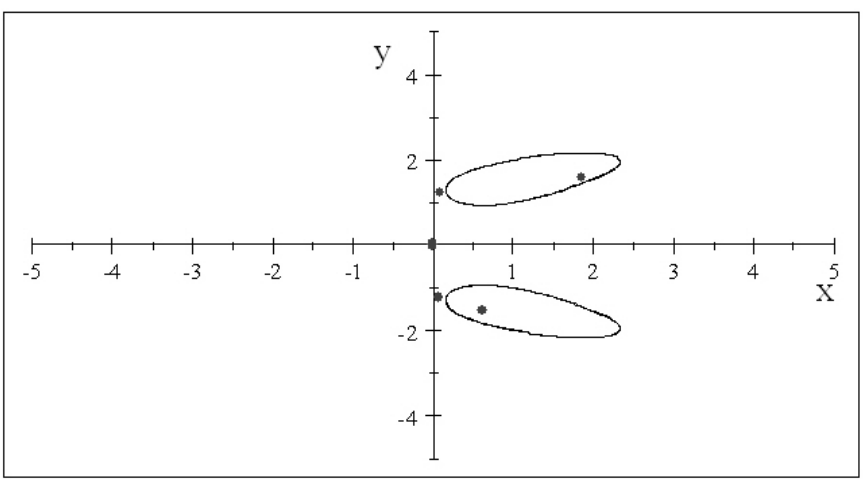

Figure 5.2: Limit cycles of system(5.2) with singular points

Example 5.2. Let $m=2, n=1, a=3, b=-2, c=1, d=-3, f=-2, h=3, P(x)=x^{3}, Q(y)=y^{3}, V(y)=-1, W(x)=1$

$$
\left\{\begin{array}{c}
\dot{x}=x\left(3 x^{5} y-2 x^{4} y^{3}-2 x^{4} y+x^{3} y^{5}-3 x^{3} y^{3}+3 x^{3} y-3 x^{2}+6 x y^{2}+2 x-5 y^{4}+9 y^{2}-3\right) \\
\dot{y}=y\left(3 x^{3} y^{3}-2 x^{2} y^{5}-2 x^{2} y^{3}+9 x^{2}+x y^{7}-3 x y^{5}+3 x y^{3}-4 x y^{2}-4 x+y^{4}-3 y^{2}+3\right)
\end{array}\right.
$$

The system (5.2) admits two limit cycles represented by the curve $3 x^{2}-2 x+y^{4}-3 y^{2}-2 x y^{2}+3=0$, and it has five singular points, three are saddle points, one is a stable focus, one is an unstable focus, the limit cycle in first quadrant encloses a stable focus, and the other encloses an instable focus. Figure (5.2)

Example 5.3. Let $m=n=1, a=2, b=-3, c=1, d=-3, f=-1, h=3, P(x)=\exp (x), Q(y)=\arctan (y), V(y)=y \sin (y), W(x)=$ $x \cos (x)$

$$
\left\{\begin{array}{c}
\dot{x}=x\left((y \exp (x)+y \sin (y))\left(2 x^{2}-3 x+y^{2}-3 y-x y+3\right)-y(2 y-x-3)\right) \\
\dot{y}=y\left((x \arctan (y)+x \cos (x))\left(2 x^{2}-3 x+y^{2}-3 y-x y+3\right)+x(4 x-y-3)\right)
\end{array}\right.
$$

The system (5.3) admits one limit cycle, represented by the curve $2 x^{2}-3 x+y^{2}-3 y-x y+3=0$, that enclosed a stable focus, figure (5.3). Note that $\frac{d(\exp (x))}{d x}+\frac{d(\arctan (y))}{d y}=e^{x}+\frac{1}{y^{2}+1}>0$

and $\int_{0}^{T} K(x, y) d t=\int_{\frac{9}{7}-\frac{2}{7} \sqrt{15}}^{\frac{2}{7} \sqrt{15}+\frac{9}{7}} \int_{\frac{1}{2} x-\frac{1}{2} \sqrt{-7 x^{2}+18 x-3}+\frac{1}{2}}^{\frac{1}{2} \sqrt{-7 x^{2}+18 x-3}}\left(e^{x}+\frac{1}{y^{2}+1}\right) d x d y \simeq 22.55$.

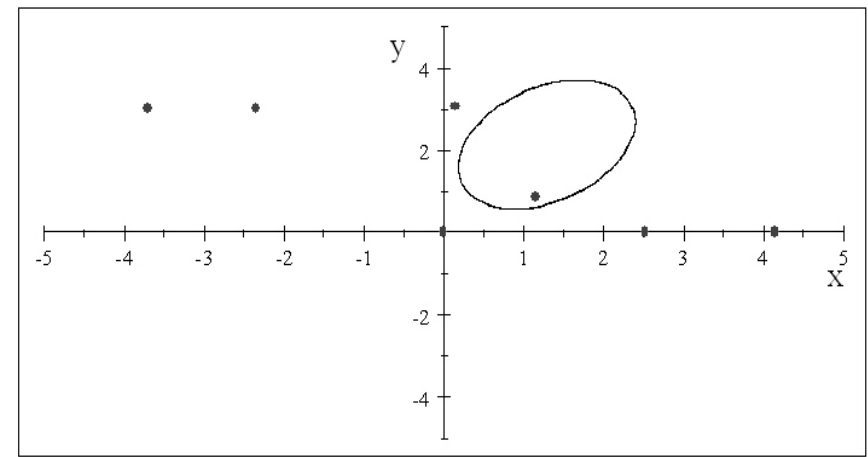

Figure 5.3: Limit cycles of system(5.3) with singular points

\section{Conclusion}

We proposed in this paper a class of Kolmogorov system, where just choose the parameters satisfying the conditions of Theorem 3.1, we conclude directly that the system has one, two or four limit cycles and we give them explicitly. 


\section{References}

[1] A. Bendjeddou and R. Cheurfa, On the exact limit cycle for some class of planar differential systems, Nonlinear differ. equ. appl. 14 (2007), $491-498$.

[2] A. Bendjeddou and R. Cheurfa, Cubic and quartic planar differential systems with exact algebraic limit cycles, Elect. J. of Diff. Equ., no15 (2011), 1-12.

[3] S. Benyoucef, A. Barbach, and A. Bendjeddou, A class of Differential system with at most four limit cycles, Annals of applied mathematics, 31 , no 4, $2015,1-9$.

[4] S. Benyoucef and A. Bendjeddou, A class of Kolmogorov system with exact algebraic limit cycles, Int.J.of Pure and Applied Mathematics, V103 no 3 , $2015,439-451$.

[5] Shen Boqian and Liu Demeng. Existence of limit cycles for a cubic Kolmogorov system with a hyperbolic solution. Northwest Math.16(1), 2000, 91-95.

[6] Cheng K.S, Uniqueness of a limit cycle for a predator-prey system, SIAM J. Math. Anal, 12 (4) (1981),541-548.

[7] SI Chengbin, Shen Boqian. The existence of limit cycles for the Kolmogorov cubic system with a quartic curve solution.J.Sys. Sci.\& Math. Scis.28(3) (2008), 334-339.

[8] H. Giacomini, M. Grau, On the stability of limit cycles for planar differential systems, J. of Diff. Equ, v 213 issue 2, 2005, 368-388.

[9] Xun C. Huang and Lemin Zhu, Limit cycles in a general Kolmogorov model, Nonlin. Anal. Theo. Meth. and Appl. 60 (2005), $1393-1414$.

[10] Huang X.C, Limit cycle in a Kolmogorov-type model, Internat. J. Math. \& Math Sci.vol 13 no 3 (1990) 555-566.

[11] X. Huang, Y. Wang, A. Cheng, Limit cycles in a cubic predator-prey differential system, J. Korean Math. Soc. 43 no 4 (2006) $829-843$.

[12] Y. Kuang and H.I Freedman, Uniqueness of limit cycles in Gause-type models of Predator-prey systems, Math. Biosci.. 88 (1988), 67-84.

[13] N. G. Lloyd, J. M. Pearson, E Sáez, I. Szántó, Limit cycles of a Cubic Kolmogorov System, Appl. Math. Lett. vol 9 no1, (1996) pp 15-18.

[14] N. G. Lloyd, J. M. Pearson, E. Sáez, and I. Szántó, A cubic Kolmogorov system with six limit cycles, International Journal Computers and Mathematics with Applications 44 (2002), 445-455.

[15] R.M May, Limit cycles in predator-prey communities, Science 177 (1972), 900-902.

[16] L. Perko, Differential equations and dynamical systems, Third edition. Texts in Applied Mathematics, 7. Springer-Verlag, New York, 2001.

[17] Peng Yue-hui. Limit Cycles in a Class of Kolmogorov Model with Two Positive equilibrium Points. Natural Science journal of Xiangtan University, Vol. 32 No.4 Dec.2010, 10-15. 